Original Research

\title{
Effects of Jugular Vein Occlusion on Cardiovascular Parameters in Horses During Exercise on a Treadmill
}

\author{
Deborah Penteado Martins Dias DVM, PhD ${ }^{\mathrm{a}}$, Marco Augusto Giannoccaro da Silva DVM, PhD ${ }^{\mathrm{b}}$, \\ Raquel Mincarelli Albernaz DVM, PhD ${ }^{\mathrm{c}}$, Lina Maria Wehrle Gomide DVM, PhD ${ }^{\mathrm{a}}$, \\ Carla Braga Martins DVM, PhD ${ }^{\mathrm{d}}$, Nara Saraiva Bernardi DVM ${ }^{\mathrm{a}}$, José Carlos Barbosa PhD ${ }^{\mathrm{e}}$, \\ Antonio de Queiroz Neto DVM, PhD ${ }^{\mathrm{c}}$, José Corrêa de Lacerda Neto DVM, PhD ${ }^{\mathrm{a}}$ \\ a Departamento de Clínica e Cirurgia Veterinária, Faculdade de Ciências Agrárias e Veterinárias-FCAV/Unesp, Jaboticabal, São Paulo, Brazil \\ ${ }^{\mathrm{b}}$ Escola de Medicina Veterinária e Zootecnia, Universidade Federal do Tocantins-UFT, Araguaína, Tocantins, Brazil \\ ${ }^{\mathrm{c}}$ Departamento de Morfologia e Fisiologia Animal, Faculdade de Ciências Agrárias e Veterinárias-FCAV/Unesp, Jaboticabal, São Paulo, Brazil

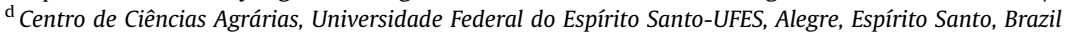 \\ e Departamento de Ciências Exatas, Faculdade de Ciências Agrárias e Veterinárias-FCAV/Unesp, Jaboticabal, São Paulo, Brazil
}

\section{A R T I C L E I N F O}

\section{Article history:}

Received 21 July 2012

Received in revised form

18 November 2012

Accepted 13 December 2012

Available online 16 February 2013

\section{Keywords:}

Equine

Exercise

Hemodynamic

Thrombophlebitis

Treadmill

\begin{abstract}
A B S T R A C T
The purpose of the present investigation was to examine the effects of unilateral and bilateral jugular vein occlusion by temporary surgical ligature on the heart rate and arterial and venous blood pressure in sedentary horses during progressive treadmill exercise. Six horses performed three exercise tests (ET). ET1, considered the control, was performed in horses without jugular occlusions. ET2 and ET3 were performed with unilateral and bilateral occlusion by temporary surgical ligature of the jugular veins, respectively. Heart rate, arterial pressure, and pressure of the occluded jugular vein were evaluated. Clinically, the horses presented apathy, head edema, congested mucous membranes, increased capillary refill time, and dysphagia. These signs were observed with the unilateral jugular vein occlusion and became more evident with the bilateral occlusion. Comparing ETs, no differences were observed in heart rate. However, jugular occlusions promoted a decrease in the mean arterial pressure and a severe increase in jugular pressure. Head edema caused by the jugular vein occlusion in the horses could interfere with the autonomic cardiovascular regulation of arterial blood pressure during exercise, likely leading to an impairment of tissue perfusion. Jugular occlusion, even unilateral, also causes severe head venous congestion, leading to venous hypertension that was aggravated by exercise, which could risk development of cerebral edema and neurological damage. The present results obtained from sedentary horses are preliminary data that lead us to suggest that sport horses presenting jugular occlusive thrombophlebitis, even unilateral, may be prevented from performing athletic activities.
\end{abstract}

(c) 2013 Elsevier Inc. All rights reserved.

\section{Introduction}

Thrombophlebitis affects the jugular veins in horses because these veins are the most commonly used sites to access venous circulation. The disease is associated

Corresponding author at: Deborah Penteado Martins Dias, Departamento de Clínica e Cirurgia Veterinária, FCAV/Unesp, Via de acesso Prof. Paulo Donato Castellane, s/n Jaboticabal, SP, 14884-900, Brazil.

E-mail addresses: dpmdias@fcav.unesp.br, deborah_dias@hotmail.com (D.P.M. Dias). with poor venipuncture technique, prolonged or improper placement of indwelling catheters, and injections of irritating drugs that lead to a mechanical or chemical injury of the vessel wall [1-8]. The clotting mechanism in horses is known to differ from that in humans, mainly reflected in a considerably shorter clotting time due to a great tendency for red blood cell aggregation [9]. Therefore, it may be presumed that such differences in clotting profile affect the reaction of the organism to catheter types and/ or materials. 
Once jugular thrombophlebitis develops, blood flow is impaired due to a reduction in the vascular lumen by partial or complete thrombus occlusion [10]. This thrombus may evolve into complete recanalization of the vein, thrombus fibrous organization without recanalization or collateral circulation $[7,11,12]$. If the jugular thrombotic occlusion persists and collateral vascularization does not occur, especially when both jugular veins are involved, the resulting dysphagia and dyspnea due to the impaired venous return of the head will likely limit athletic performance $[4,10,13]$.

To ensure a proper perfusion of active muscles during exercise, the cardiovascular system must maintain the blood pressure within narrow limits. Neural (sympathetic stimulation) and endocrine (release of catecholamines, activation of the renin-angiotensin-aldosterone system and stimulation of vasopressin release) mechanisms are activated to maintain vascular tone by preserving the blood volume [14]. In the athletic horse, this ability is well developed, and, due to increments in heart rate (HR) and arterial pressure, the cardiac output resting value may be eightfold increased during maximal exercise $[15,16]$. Therefore, cardiovascular regulation during physical exercise is characterized by transitory adjustments that are considered similar in trained and untrained individuals and could change as a consequence of the conditioning of the cardiovascular system in response to training $[17,18]$. However, many cardiovascular disorders are known to result in hemodynamic disarrangements that can interfere with this adaptive response and result in inadequate tissue perfusion.

Exercise and training are known to have considerable effects on mechanisms of blood coagulation in horses, leading to increased platelet aggregation and increased fibrinolytic activity [19-21]. However, the few studies that have been conducted in horses demonstrating coagulation parameter shifts due to exercise performance did not evaluate horses presenting thrombotic diseases as jugular thrombophlebitis.

In an attempt to correlate the presence of jugular thrombophlebitis in horses with their athletic performance, Moreau and Lavoie [7] examined the records of horses that presented the disease during their athletic lifespan. The authors suggested that thrombophlebitis did not affect the performance of horses used for leisure riding and other nonracing activities, even when the horses suffered from bilateral occlusion. However, thrombophlebitis in racing Standardbred horses was associated with a decreased chance of returning to racing. The retrospective study by Moreau and Lavoie [7] is the only report comparing the presence of jugular occlusion with performance in horses. Thus, the hemodynamic parameters in exercising horses presenting jugular vein occlusion have never been studied. Based on the hypothesis that head venous congestion due to the stoppage of jugular vein blood flow could modify cardiovascular dynamics during exercise, the purpose of the present investigation was to examine the effects of unilateral and bilateral jugular vein occlusion by temporary surgical ligature on the heart rate and blood pressure in sedentary horses during progressive exercise on a high-speed treadmill, aiming to understand the same effects in the athletic horse presenting jugular thrombophlebitis.

\section{Materials and Methods}

\subsection{Horses and Study Design}

Research trials were performed with six sedentary mixed breed adult horses, three geldings, and three mares with a mean age of $6 \pm 1$ years and a mean body weight of $344 \pm 12 \mathrm{~kg}$. All horses were considered healthy based on physical examination and blood count (PocH-100iV Diff; Sysmex, Brazil). During the experimental period, horses were housed in individual stalls and consistently fed concentrated pelleted feed and hay (Cynodon dactylon), twice a day with 12-hour intervals. Water was provided ad libitum. The six horses performed three sessions of an incremental exercise test (ET) on a high-speed treadmill (Galloper; Sahinco, Palmital, Brazil) housed in an airconditioned laboratory set at $25^{\circ} \mathrm{C}$, with a 24-hour interval between sessions. The first test (ET1), considered the control, was performed in horses without jugular vein occlusion. The second (ET2) and third (ET3) tests were performed in horses with occlusion by ligature of the left jugular vein and with occlusion of both jugular veins, respectively. All experimental protocols performed in the current study were approved and supervised by the institutional committee on animal research and ethics (protocol no. 004302-06).

\subsection{Treadmill Exercise Protocols}

One week before the exercise tests, the horses were acclimated to the treadmill over 3 days. On the first day, they were led onto and stopped on the equipment several times to acquaint them with the room, rubber floor, and side and front safety bars. After they adjusted to this procedure, they were fitted with a safety belt, walked (1.5 $\mathrm{m} / \mathrm{s}$ ) on the treadmill for 5 minutes and then stopped. This procedure was repeated three times. On the second day, the horses were led onto the equipment, walked $(1.5 \mathrm{~m} / \mathrm{s})$ for 5 minutes and trotted $(3.5 \mathrm{~m} / \mathrm{s}, 4.5 \mathrm{~m} / \mathrm{s}$, and $5.5 \mathrm{~m} / \mathrm{s})$ for 3 min at each speed. Some horses adjusted quickly to the treadmill and could be cantered $(6.5 \mathrm{~m} / \mathrm{s}, 7.5 \mathrm{~m} / \mathrm{s}$, and 8.5 $\mathrm{m} / \mathrm{s}$ ) for $2 \mathrm{~min}$ at each speed on the same day. On the third day, the procedure was repeated, with all horses being walked, trotted, and cantered.

The total duration of each ET was $83 \mathrm{~min}$, including 18 min of incremental exercise, rigorously timed according to a predetermined rest and exercise sequence (Table 1 ). The proposed exercise test could be considered medium intensity. Finally, the horses cooled down with $5 \mathrm{~min}$ of trotting and 10 min of walking, followed by 30 minutes of recovery at rest. The entire test was performed on a level surface. The cardiovascular variables were recorded at 13 time points ( $T$ ). The first evaluation (T0) was performed at rest at the last 30 seconds before the start of the exercise. During exercise, measurements were taken at the last 30 seconds before each speed change (T1-T10). During the recovery phase, two measurements were taken at rest 15 minutes (T11) and 30 minutes (T12) after the end of the exercise. 
Table 1

Treadmill exercise test (ET) protocol performed by horses subjected to treadmill ETs without jugular vein occlusion (ET1), with unilateral jugular vein occlusion (ET2), and with bilateral jugular vein occlusion (ET3)

\begin{tabular}{|c|c|c|c|c|c|}
\hline \multirow{2}{*}{$\begin{array}{l}\text { Condition } \\
\text { Rest }\end{array}$} & \multirow{2}{*}{$\begin{array}{l}\begin{array}{l}\text { Duration } \\
(\mathrm{min})\end{array} \\
5 \mathrm{~min}\end{array}$} & \multirow{2}{*}{$\begin{array}{l}\text { Speed } \\
(\mathrm{m} / \mathrm{s})\end{array}$} & \multicolumn{2}{|c|}{$\begin{array}{l}\text { Measurements } \\
\text { At }\end{array}$} & \multirow{2}{*}{$\begin{array}{l}\text { Cumulative } \\
\text { Time (min) } \\
5\end{array}$} \\
\hline & & & T0 & $4 \mathrm{~min}$ & \\
\hline \multirow[t]{2}{*}{ Warm-up } & 10 & 1.7 & $\mathrm{~T} 1$ & $14 \mathrm{~min} 30 \mathrm{~s}$ & 15 \\
\hline & 5 & 3.5 & $\mathrm{~T} 2$ & $19 \min 30 \mathrm{~s}$ & 20 \\
\hline Progressive & 3 & 4.5 & T3 & $22 \min 30 \mathrm{~s}$ & 23 \\
\hline \multirow[t]{5}{*}{ exercise } & 3 & 5.5 & $\mathrm{~T} 4$ & $25 \min 30 \mathrm{~s}$ & 26 \\
\hline & 3 & 6.5 & T5 & $28 \mathrm{~min} 30 \mathrm{~s}$ & 29 \\
\hline & 3 & 7.5 & T6 & $31 \mathrm{~min} 30 \mathrm{~s}$ & 32 \\
\hline & 3 & 8.5 & $\mathrm{~T} 7$ & $34 \mathrm{~min} 30 \mathrm{~s}$ & 35 \\
\hline & 3 & 9.5 & T8 & $37 \mathrm{~min} 30 \mathrm{~s}$ & 38 \\
\hline \multirow[t]{2}{*}{ Cool-down } & 5 & 3.5 & T9 & $42 \min 30 \mathrm{~s}$ & 43 \\
\hline & 10 & 1.7 & $\mathrm{~T} 10$ & $52 \min 30 \mathrm{~s}$ & 53 \\
\hline \multirow[t]{2}{*}{ Rest } & 30 & 0 & $\mathrm{~T} 11$ & $68 \mathrm{~min}$ & 68 \\
\hline & & & $\mathrm{T} 12$ & $83 \mathrm{~min}$ & 83 \\
\hline
\end{tabular}

\subsection{Cardiovascular Evaluation}

HR was measured in beats per min (bpm) using a HR monitor (S810i heart rate monitor; Polar Electro Oy, Kempele, Finland), with the electrodes placed in close contact with the horse's skin surface by using coupling gel. The local pressure $(\mathrm{mmHg})$ of the left jugular vein was also evaluated and referred to as the regional jugular vein pressure (RJVP) in this study. Measurement was taken through a catheter (14-gauge $\times 1.75$-in Insyte; Becton Dickinson, Juiz de Fora, Brazil) implanted in the left jugular vein (cranial third) against its blood flow direction. Implantation was performed as usual, immediately before ET1. The catheter was sutured to the skin and flushed with heparinized saline solution $(10 \mathrm{U} / \mathrm{mL})$ every 8 hours until the end of ET3, when it was removed. The left jugular vein occlusion by ligature was performed $15 \mathrm{~cm}$ caudal to the catheter implantation site, so that in ET2 and ET3, the RJVP was recorded from the blood flow arriving in the cranial vessel segment above the ligature. The systolic arterial pressure (SAP), diastolic arterial pressure (DAP) and mean arterial pressure (MAP) were also measured $(\mathrm{mmHg})$ during the three exercise tests. For this purpose, a $20-\mathrm{cm}$ polyurethane catheter (Central Venous Catheterization set, ref CV-04701; Arrow International, Reading, PA) was implanted in the left facial artery [22]. The arterial line was flushed every 8 hours with a 10\% ascorbic acid solution containing $5000 \mathrm{U}$ of sodium heparin per $\mathrm{mL}$ [22] until the end of ET3, when it was removed. The arterial catheter implantation involved a minor surgical procedure, which was performed $24 \mathrm{~h}$ before ET1 to exclude the cardiovascular effects of acepromazine and pethidine chloride. Immediately before each exercise session, the horse was led onto the treadmill, and the catheters implanted into the left jugular vein and left facial artery were connected to transducers by $2 \mathrm{~m}$ long tubes filled with heparinized saline solution $(10 \mathrm{U} / \mathrm{mL})$. Figure 1 shows one horse just before ET1. The transducers were placed firmly on a stable bar of the equipment at the level of the point of the horse's left shoulder and calibrated. Blood pressure data were displayed on a multiparametric monitor (model DX-2010; Dixtal, São Paulo, Brazil). Cardiovascular parameter measurements were recorded during ET1, ET2, and ET3 at 13 time points (T0-T12) per test.

\subsection{Jugular Vein Occlusion}

The left jugular vein was occluded by ligature 12 hours before ET2. Therefore, at ET2, the horses were evaluated after 12 hours of unilateral blood flow occlusion. After ET2, the left jugular vein was kept occluded, and the right jugular vein was ligatured 12 hours before ET3. Therefore, at ET3 the horses were evaluated after 12 hours of bilateral blood flow occlusion. The ligature procedure was performed with horses that were unsedated and restrained in stocks. For each left and right jugular vein occlusion, the same procedure was performed. The hair was clipped over the lateral aspect of the neck, followed by an infiltration of $5 \mathrm{~mL}$ of $2 \%$ lidocaine chloride into the

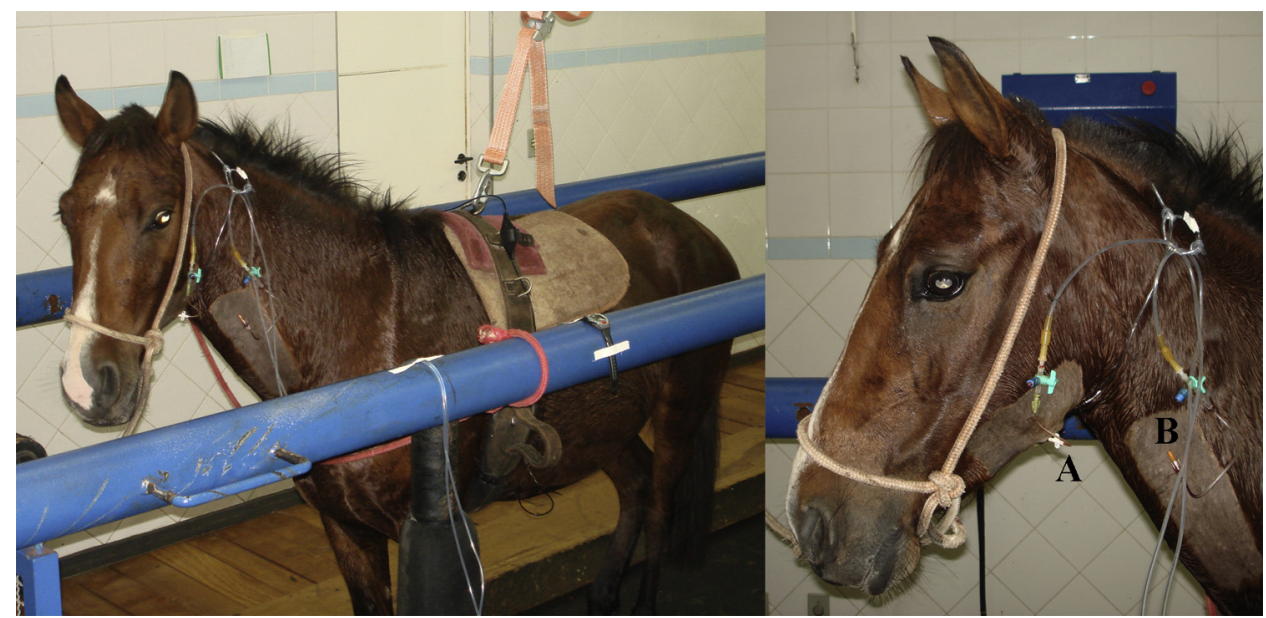

Fig. 1. Horse properly implanted with a catheter in the left facial artery to measure arterial pressure (A) and in the left jugular vein to measure regional jugular vein pressure (B), shown standing on the treadmill just before the first exercise test. Proper placement of the treadmill safety belt and heart rate monitor also can be also observed. 
subcutaneous tissue. After sterile preparation, a $5-\mathrm{cm}$ skin incision was made in a craniocaudal direction above the middle third of the jugular groove. The incision was deepened by blunt dissection, and the jugular vein was located. The vein was isolated by one Penrose drain passed around the vessel using a curved Kelly forceps, and the same drain was used to carry out the complete occlusive ligature of the jugular vein. Meticulous hemostasis was required to avoid postoperative hematoma or seroma formation. The skin was closed using nylon 1 in a simple interrupted pattern. Immediately after ET3, the sutures applied at both sides were opened, and the ligatures were released. After confirmation that blood flow was restored, the subcutaneous tissue was sutured using polyglactin 910 suture (Vycril 0; Ethicon Johnson \& Johnson, São José dos Campos, Brazil) in a Cushing pattern. The skin was closed using nylon 1 in a simple interrupted pattern. Starting with the arterial catheter implantation, all horses were administered procaine penicillin $\mathrm{G}$ at $22,000 \mathrm{U} / \mathrm{kg} \mathrm{IM} \mathrm{q} \mathrm{for} 12 \mathrm{~h}$ for 8 days and flunixin meglumine at $0.5 \mathrm{mg} / \mathrm{kg} \mathrm{IM} \mathrm{q} \mathrm{for} 12 \mathrm{~h}$ for 8 days. Daily dressing of the suture lines with $1 \%$ povidoneiodine solution was performed until the retirement of the animals, when the sutures at the site of the facial artery catheter implantation and jugular vein occlusions were permanently removed.

\subsection{Clinical Evaluation}

The horses were clinically evaluated on the first experimental day just before the initial contact with the treadmill and then daily from the facial artery catheter implantation until the retirement of the animals. Their behavior, water and food intake, intestinal motility, HR (bpm), respiratory rate measured in breaths per min (brpm), breathing pattern, rectal temperature, mucous membrane color, and capillary refill time (seconds) were assessed. The presence of head edema related to jugular vein occlusion was also evaluated. The scores were based on the statistical assessment of nonparametric data. Behavior was considered normal (0), mildly apathetic (1), moderately apathetic (2), or severely apathetic (3). Water and food intake was considered normal (0), mildly dysphagic (1), moderately dysphagic (2), or severely dysphagic (3). Intestinal motility and breathing pattern were considered normal $(0)$ or abnormal (1). Mucous membranes were considered normal $(0)$, slightly congested (1), moderately congested (2), or severely congested (3). Finally, head edema was considered absent (0), mild (1), moderate (2), or severe (3).

\subsection{Statistics}

The nonparametric data collected in the clinical evaluations were converted to scores that could be compared. Values were analyzed by analysis of variance (ANOVA), and means were compared by Tukey's test by using AgroStat version 1.1.0.626/2011 software (Jaboticabal, Brazil). A significant difference between means was considered to be a $P$ value of $<.05$ for scores and a $P$ value of $<.01$ for the cardiovascular variable data obtained during the exercise tests. Data are shown as means \pm standard error of the means (SEM).

To better understand the methods, Figure 2 presents a time line in days (D), including all events performed during the experimental period for each horse.

\section{Results}

\subsection{Clinical Findings}

All horses were considered healthy based on clinical findings and blood count measured on day 1 (D1). Physical examination showed a $\mathrm{HR}$ of $40 \pm 2 \mathrm{bpm}$, respiratory rate of $12 \pm 1 \mathrm{brpm}$, and rectal temperature of $37.8 \pm 0.1^{\circ} \mathrm{C}$. The mucous membranes had a normal pink color, and capillary refill time was 2 seconds. Food and water intake and intestinal motility were also normal. The horses were alert and did not present signs of lameness. Blood count revealed red blood cell concentrations of $5.6 \times 10^{6} / \mu \mathrm{L} \pm$ $0.6 \times 10^{6} / \mu \mathrm{L}$; hemoglobin of $9.9 \mathrm{~g} / \mathrm{dL} \pm 0.3 \mathrm{~g} / \mathrm{dL}$; packed cell volume of $35.3 \% \pm 0.9 \%$; white blood cell counts were $9.9 \times 10^{3} / \mu \mathrm{L} \pm 0.4 \times 10^{3} / \mu \mathrm{L}$, and platelet counts were 184 $\times 10^{3} / \mu \mathrm{L} \pm 5 \times 10^{3} / \mu \mathrm{L}$.

Differences in clinical findings were observed on D10 when the left jugular vein was occluded and on D11 when both jugular veins were occluded. The horses showed mild to moderate apathy on D10 and moderate to severe apathy on D11. The left unilateral occlusion (D10) leads to the development of edema located ventral to the jaw and at the left parotid region, which was observed as mild in three horses and severe in the other three horses. The bilateral occlusion leads to generalized head edema, especially located ventral to the jaw, left and right masseter muscles, parotid regions, lips, and tongue. In all horses, the mucous

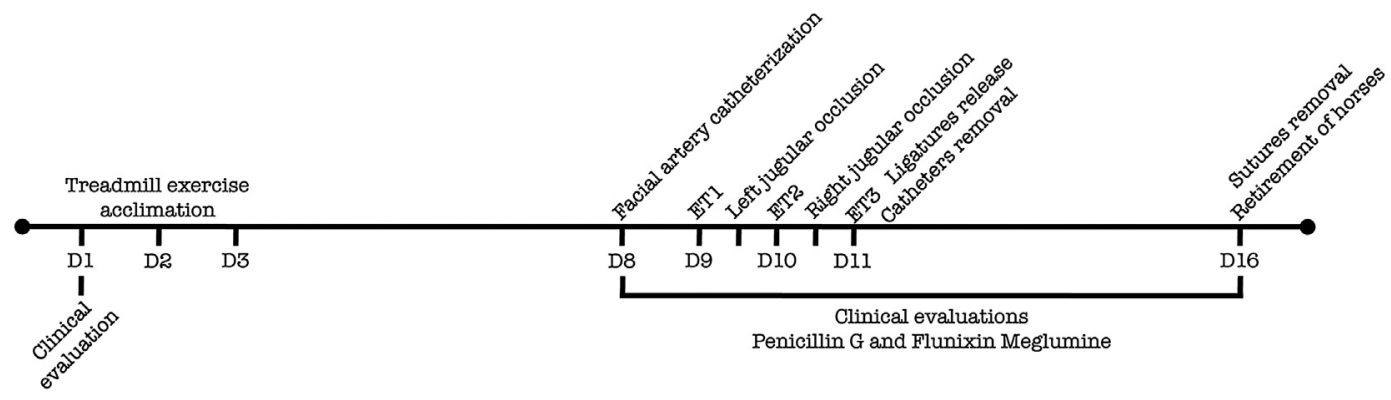

Fig. 2. Time line in days (D) showing all events performed during the experimental period for each horse $(\mathrm{n}=6)$. 
membranes had a light red color and were slightly congested on D10 and moderately congested on D11. These changes were followed by a capillary refill time of 2 to 3 seconds on D10 and 3 to 4 seconds on D11. On D10, three horses showed mild dysphagia, which became severe on D11 when all horses presented prehension, mastication, and swallowing distress. Despite these alterations, their water and food intake, intestinal motility, HR, respiratory rate, breathing pattern, and rectal temperature remained unchanged.

\subsection{Exercise Test and Cardiovascular Changes}

The incremental exercise tests were performed without complications, and HR, SAP, DAP, MAP, and RJVP records were obtained as proposed. Individually in each ET, the HR measurements performed at rest showed no differences between the time points before (T0) and after (T11-T12) the exercise. The differences in the HR were observed with exercise progression, comparing the measurements at rest (T0, T11, and T12) with those taken during exercise (T1T10). In addition, differences were detected among the exercise HR values (T1-T10) (Table 2). The HR increased in response to the exercise intensity, with the highest average values obtained at the time points of increased treadmill speed and effort intensity. No differences among the tests were detected (Fig. 3).

For the arterial pressure, the SAP, DAP, and MAP showed no changes among rest values (T0, T11, and T12). Individually in each ET, the same pattern was observed for the three tests during exercise (T1-T10), with an increased SAP, a decreased DAP, and a nearly unchanged MAP following the speed and effort increments. The response of the arterial blood pressure to the progressive exercise in the healthy horses (ET1) is illustrated in Figure 4. Differences in MAP were detected only within ET1, with the MAP values obtained at maximal effort (T8) being higher than the average values obtained during the walking exercise phases (T1 and T10) and at rest after the exercise (T11 and T12) (Table 2). However, more differences among the time points were observed within the same ET for the SAP and DAP in the tests performed with unilateral and bilateral jugular occlusion (ET2 and ET3) (Table 2). Compared with T0 (rest), the SAP increased at T5 and T8 within ET1, from T4 to T9 within ET2, and from T6 to T9 within ET3. Compared with T0 (rest), the DAP decreased from T5 to T7 within ET1, at T1 and from T3 to T9 within ET2, and from T3 to T10 within ET3. These results showed that the SAP and DAP changes were more evident with jugular occlusions, extending to the warm-up and cool down exercise phases in ET2 and extending to cool down in ET3. Considering the changes due to jugular occlusion, the SAP showed differences only at T7 (Fig. 5A). The DAP showed differences at two time points of maximal effort, decreasing at T7 and T8 (Fig. 5B). The most evident effects of the jugular vein occlusions involved the decrease in MAP. From T4 to T7, MAP decreased in ET3 (Fig. 5C).

Individually in each ET, the RJVP showed differences only within the tests performed with jugular vein occlusion (ET2 and ET3). The RJVP increased at T8 compared with the time at rest (T0, T11 and T12) within ET2 and increased from T4 to T8 compared with the time at rest after the

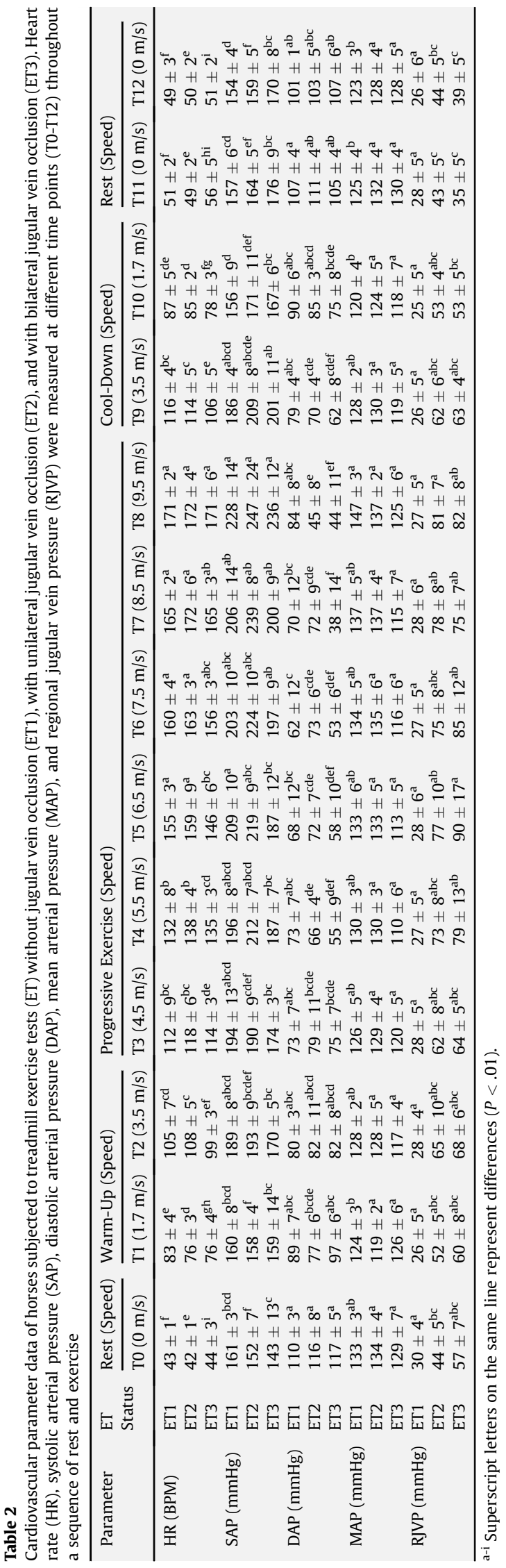




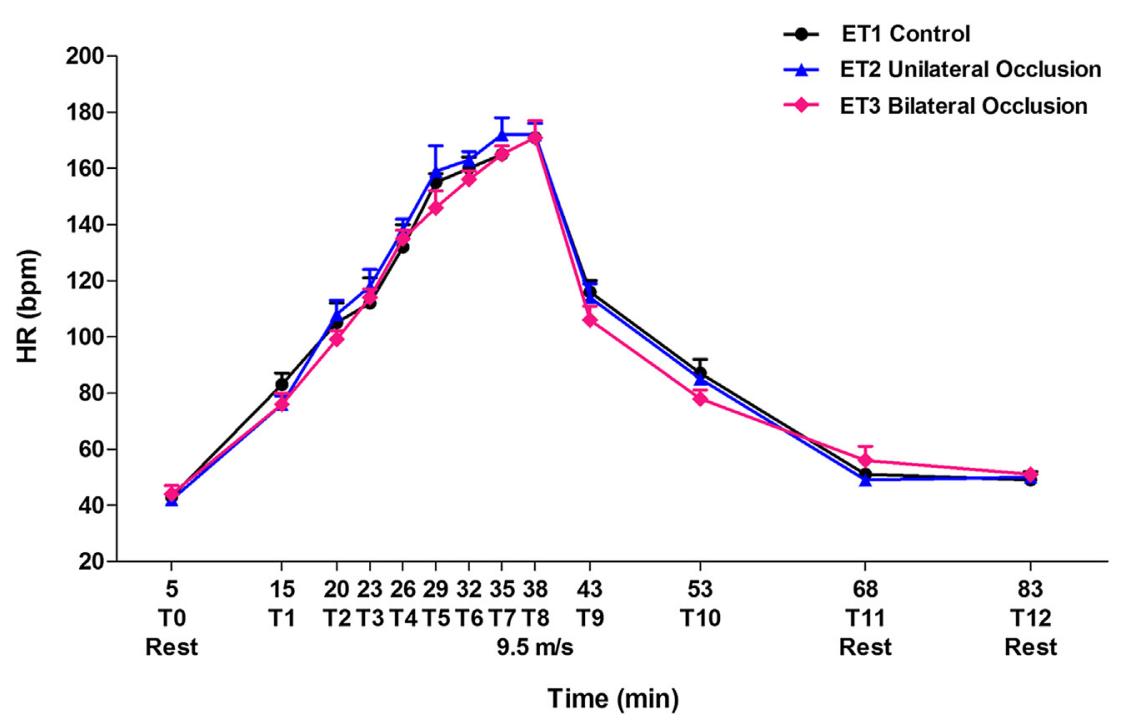

Fig. 3. Mean heart rate (HR) of horses $(n=6)$ during the treadmill exercise test (ET) without jugular vein occlusion (ET1), with unilateral jugular vein occlusion (ET2), and with bilateral jugular vein occlusion (ET3). The HR (BPM) was measured at different time points (T0-T12) throughout a sequence of rest and exercise.

exercise (T11 and T12) within ET3 (Table 2). The bilateral jugular vein occlusion at ET3 increased the RJVP values at T0 (rest) compared with ET1. The most evident differences were detected with exercise progression, when RJVP increased in ET2 and ET3 from T1 to T10 compared with the average values obtained in ET1. The differences detected in the RJVP among the exercise tests are shown in Figure 6.

\section{Discussion}

The clinical findings observed in horses subjected to jugular blood flow occlusion, such as head edema, dysphagia, congested mucous membranes and increased capillary refill time, are related to the venous congestion that developed following the ligature. These observations are in accordance with the clinical occurrence of completely occlusive jugular thrombophlebitis $[4,10,13]$. The rectal temperature, HR, respiratory rate and breathing pattern did not show significant differences during the protocol period, suggesting that the flunixin meglumine therapy effectively provided comfort to the horses despite the three surgical procedures (facial artery catheterization and the left and right jugular vein ligatures) and the three exercise sessions performed.

HR data during exercise tests were assessed to determine the effort intensity. According to Sirotsky et al [23],

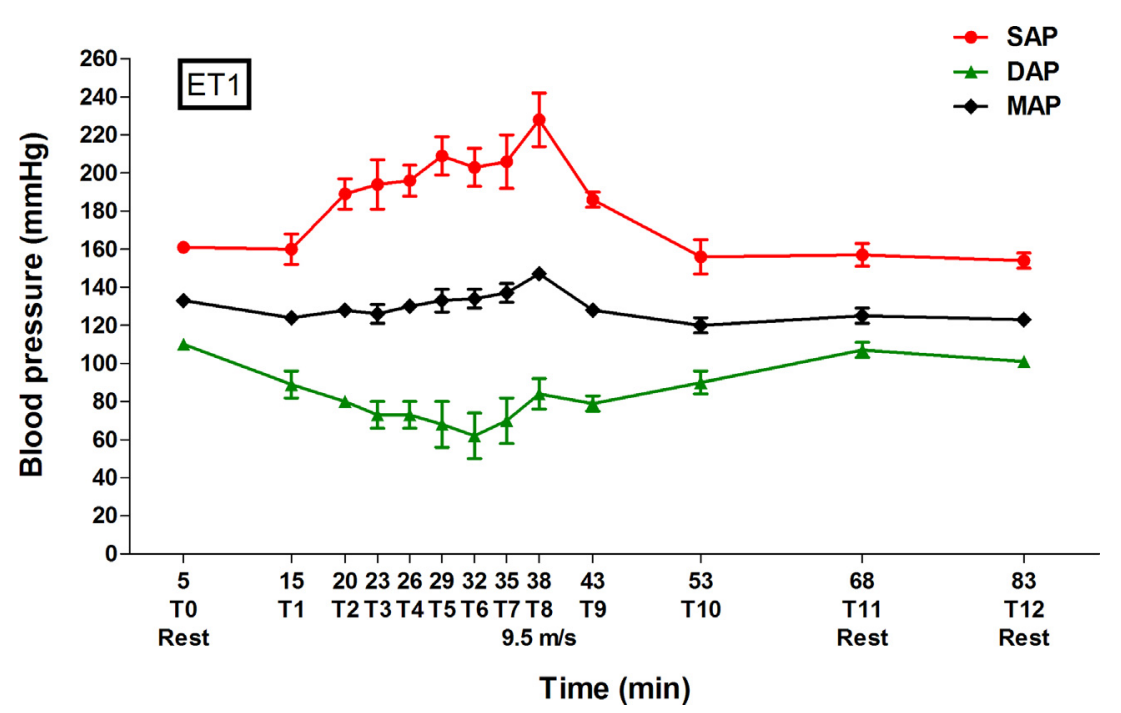

Fig. 4. Systolic arterial pressure (SAP), diastolic arterial pressure (DAP), and mean arterial pressure (MAP) responses to the progressive treadmill exercise test performed by healthy horses $(\mathrm{n}=6)$. The SAP, DAP, and MAP $(\mathrm{mm} / \mathrm{Hg})$ were measured at different of time points (T0-T12) throughout a sequence of rest and exercise. 
the maximal HR determined on a treadmill for mixed breed horses, which are similar to horses used in the present study, is $216.8 \mathrm{bpm}$. Considering that the horses presented a HR of $171 \pm 2$ in ET1, $172 \pm 4$ in ET2, and $171 \pm$ 6 in ET3 at maximal effort (T8), the exercise tests reached $80 \%$ of maximal HR, characterizing the exercise as submaximal. Furthermore, the study conducted by Sirotsky et al [23] was the only report HR values during treadmill exercise for mixed breed horses from our region, indicating the importance of reporting the present HR values.

Although the present results provide novel information regarding the cardiovascular dynamics in horses with unilateral and bilateral jugular vein occlusion, they must be interpreted cautiously due to the lack of studies of the same subject. The absence of similar studies leads us to delineate some hypotheses to discuss the observed differences. Cerebral edema develops in response to an increase in cerebral capillary pressure. Once started, the edema compresses the blood vessels, resulting in reduced blood flow and leading to cerebral ischemia that induces arteriolar dilation. This dilation increases the capillary pressure even further, aggravating the edema. Because the brain is inside a rigid cavity, the excess fluid compressing the blood vessels can lead to nervous tissue damage [14]. In humans, vasogenic edema is reported to be the most common form of cerebral edema [24]. Furthermore, the occlusion of both jugular veins increases morbidity due to cephalic venous hypertension, which results in increased cerebrospinal pressure [25]. The Queckenstedt maneuver is a clinical test used for diagnosing spinal stenosis in humans. Bilateral jugular occlusion by compression is performed, leading to an engorgement of spinal veins and a fast increase of cerebrospinal fluid pressure [26]. The methods and observations related to the Queckenstedt maneuver highlight that jugular vein occlusion may directly lead to cephalic venous hypertension, which could conduce to serious complications if occlusions are maintained. The consequences of increased cerebrospinal pressure are blindness, headaches, lethargy, convulsions, respiratory depression, coma and death [25]. Compared with other mammalian species, horses do not have a deep or internal jugular vein [27], and thus the consequences of jugular occlusion by thrombus could be considered worse. The horses in the present study developed venous congestion and head edema, which were confirmed by clinical evaluations. Assuming that the edema extends to the nervous tissue, as described for humans [25], the cardiovascular autonomic regulation could be affected, explaining the blood pressure differences among the exercise tests.

The sinoatrial node acts as the primary pulse generator for the heart beat and is controlled by the parasympathetic and sympathetic nervous systems. Under rest conditions, both branches of the autonomic nervous system are tonically active, regulating cardiac activity predominantly via the parasympathetic system. When exercise is started, the catecholamine release is immediately stimulated, and the sympathetic activity exerts a positive chronotropic effect on the heart, increasing the HR in an attempt to increase cardiac output for a better perfusion of the active tissues [18,28-31]. This cardiovascular regulation is well understood and was observed individually in each ET. The HR increased linearly throughout the exercise, showing the highest values within the high speeds of the treadmill and with increased effort. No differences were detected in the HR values among the three ET, showing that jugular occlusion did not affect the sympathetic activity with respect to the positive chronotropic effect on the heart.

During exercise, the catecholamine release activated the sympathetic nervous fibers, which also led to a positive inotropic effect on the heart, increasing the blood pressure [14]. In addition, the sympathetic stimulation on the veins leads to vasoconstriction in inactive tissues, such as the kidneys and splanchnic tissue, and the capacitance vessels are compressed by the abdominal musculature. These episodes increased the venous return to the heart. The greater volume of blood entering the heart during diastole increases the stroke volume, leading to an elevated SAP. In contrast, the DAP decreases due to the reduced total peripheral resistance to provide a better perfusion of the active muscles [29]. Blood pressure is known to be a balance between cardiac output and total peripheral resistance [14]. Actually, during exercise, the SAP increases due to an increased cardiac output, and the DAP decreases due to a decreased peripheral resistance following a progressive effort to continuously maintain the MAP at a constant level, especially during aerobic exercise. If the effort increases to a high intensity that changes the metabolic pathways to an anaerobic condition, the MAP tends to increase [32]. The present results corroborate this description of the blood pressure responses to exercise progression, and they were similar for the three ET, showing that the SAP increased, the DAP decreased and the MAP remained stable. Therefore, supported by these blood pressure results, the exercise performed can be characterized as predominantly aerobic, which is also in accordance with the obtained HR values.

The first evidence that unilateral or bilateral jugular vein occlusion interferes with blood pressure regulation during exercise were shown by results obtained individually in each ET. Compared with T0 (rest), both the SAP and DAP had more differences in the time points within the tests performed with occlusions (ET2 and ET3). Such differences were even more pronounced for the DAP results compared with the resting values (T0). ET1 showed three time points (T5-T7) whereas ET2 showed eight time points (T1, T3-T9) and ET3 showed eight time points (T3T10) with decreased DAP values. The MAP increased at T8 in ET1 compared with rest, which could be interpreted as a limited effort to change the exercise metabolism from predominantly aerobic to predominantly anaerobic, as previously described [32]. However, during ET2 and ET3, the same effort was performed, and the MAP did not change. Venous congestion, observed in cases of thrombophlebitis in horses, increases the hydrostatic pressure leading to head edema $[4,10]$. Considering that no differences were observed in the HR among the tests, the head edema appears to be due to the jugular occlusions during the occlusive period performed in the present study, which affected the sympathetic activity regarding the inotropic effects on the heart and the peripheral vasotonic effects. Furthermore, because the DAP showed more differences than the SAP, the peripheral vasotonic effects, 
A

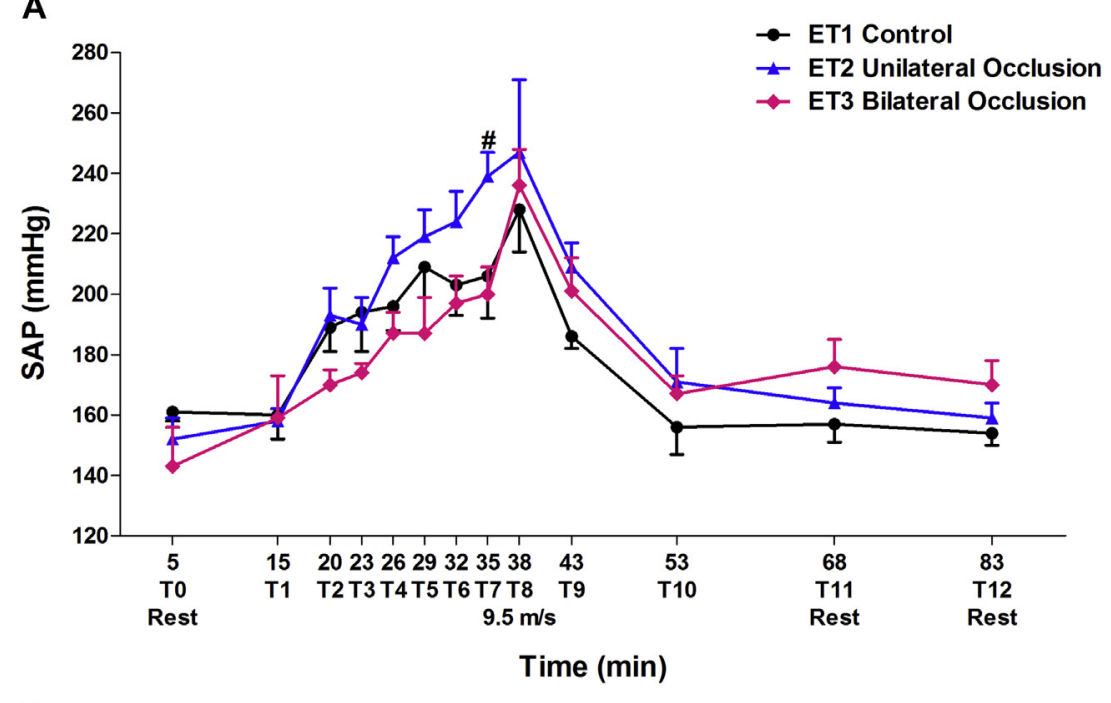

B
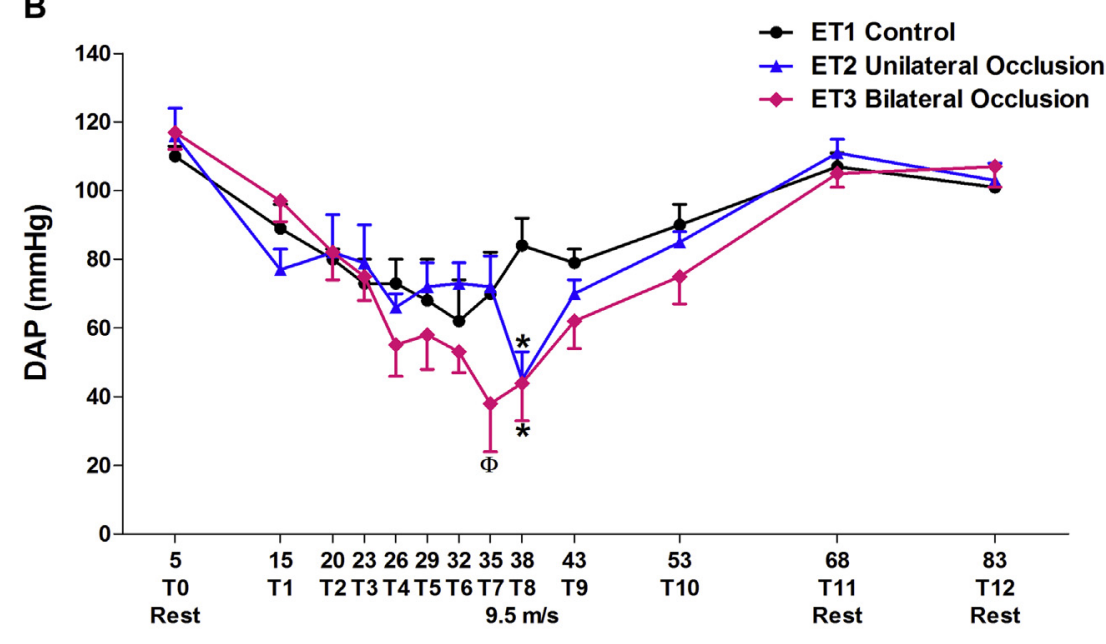

Time $(\min )$

C

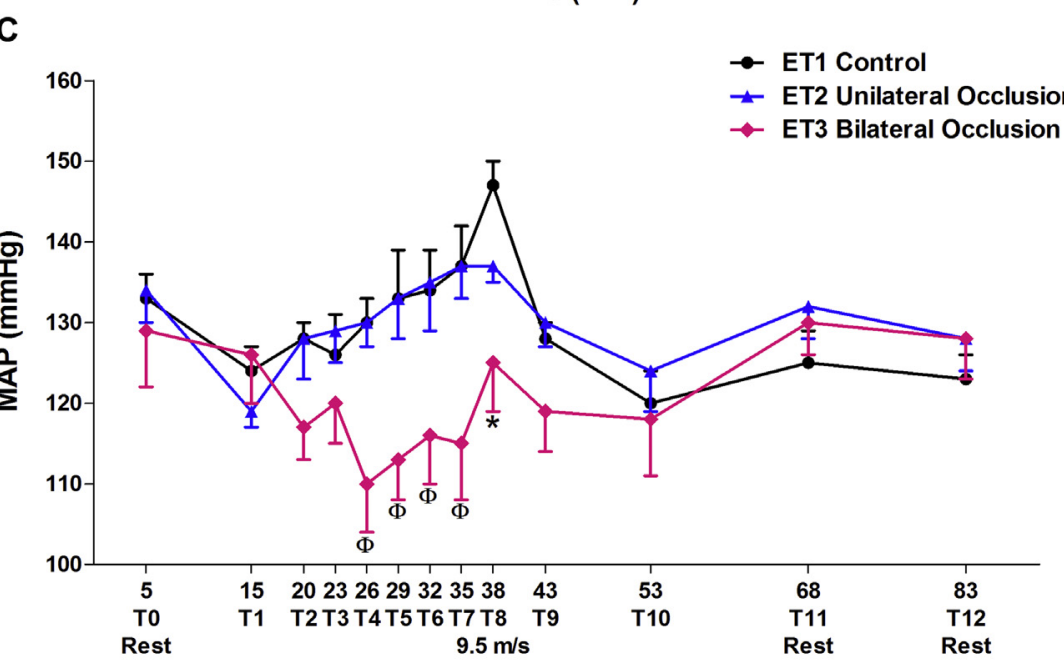

Time (min)

Fig. 5. Arterial blood pressure of horses $(n=6)$ during the treadmill exercise test (ET) without jugular vein occlusion (ET1), with unilateral jugular vein occlusion (ET2), and with bilateral jugular vein occlusion (ET3). The blood pressure ( $\mathrm{mmHg}$ ) was measured at different time points (T0-T12) throughout a sequence of rest 


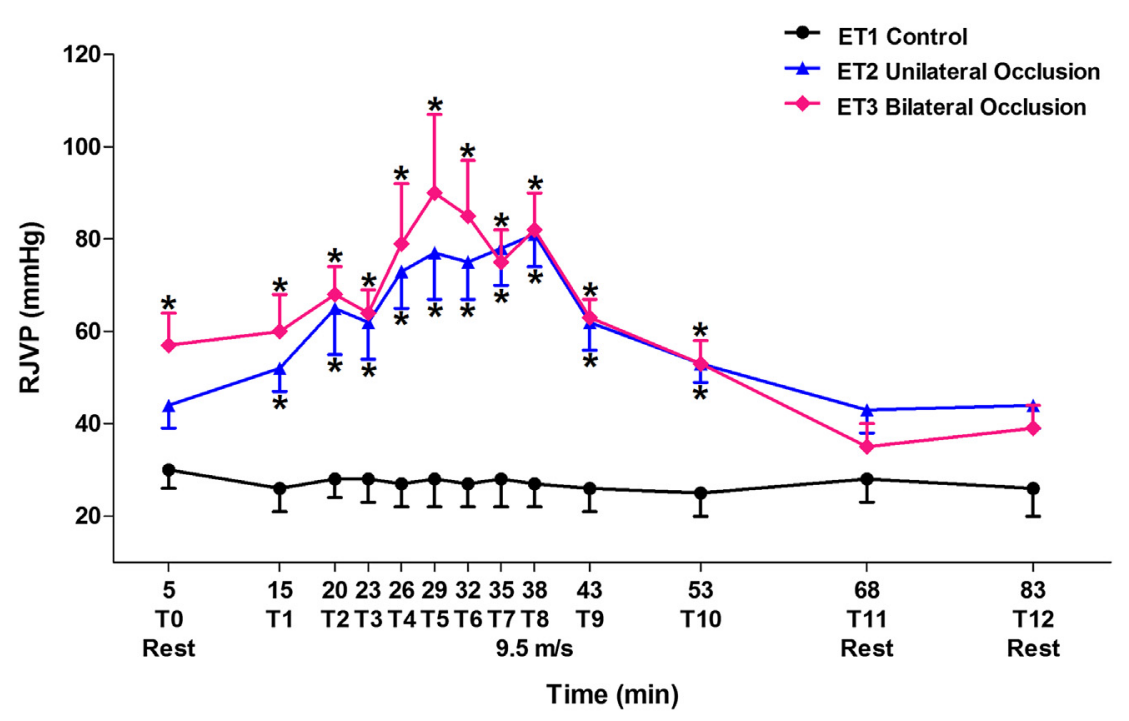

Fig. 6. Regional jugular vein pressure (RJVP) of horses $(n=6)$ during the treadmill exercise test (ET) without jugular vein occlusion (ET1), with unilateral jugular vein occlusion (ET2), and with bilateral jugular vein occlusion (ET3). RJVP (mmHg) was measured at different time points (T0-T12) throughout a sequence of rest and exercise. *Differences from ET1 $(P<.01)$.

related to DAP, were most likely more affected by the occlusions than the inotropic positive effect on the heart, related to SAP.

So far, we discussed blood pressure regulation within the tests individually. Now, comparing the same time point values among the tests, we could obtain a good sense of the effects of jugular occlusion. This discussion is also based on the same hypothesis that head edema compresses the central nervous tissue. The most marked differences were observed in the MAP values, which decreased substantially with the bilateral occlusion during the more intense exercise phase (T4-T8). Given that the MAP indicates the balance between cardiac output and total peripheral resistance [14], which should be maintained within narrow limits for adequate perfusion during physical activity [29], the decreased values obtained with bilateral occlusion could indicate poor tissue perfusion during exercise. Jugular thrombophlebitis recovery in horses is known to be followed by the development of collateral circulation [7]. Whether the collateral circulation is sufficient to supply adequate head venous return after the occurrence of bilateral occlusive jugular thrombophlebitis is unclear; the present results suggest that these horses could perform poorly due to poor muscular tissue perfusion, whereas cardiovascular responses to exercise were compromised.

With respect to RJVP, the results suggested that the jugular vein occlusion, even unilateral, promoted severe head venous congestion, leading to hypertension that was aggravated by exercise. The RJVP during ET2 and ET3 reached values two fold higher than normal (ET1) with exercise progression. The observed jugular venous hypertension during ET2 and ET3 could be attributed only to the mechanical obstruction, with no other apparent reason for the occurrence of venous hypertension. This hypertension was most likely distributed throughout the head based on the edema observed on the clinical evaluations. Considering that increased cerebral capillary pressure may further the development of cerebral edema [14], these horses risk neurological damage. This issue led us to contraindicate physical activity for those horses that had jugular thrombophlebitis and still present thrombus obstruction in the jugular vein, unless collateral circulation could be proven to have developed to completely supply the deficit of venous return from head.

\section{Conclusions}

The results of this study, performed in sedentary horses, are only preliminary data for the effects of jugular vein occlusion on cardiovascular parameters in exercising horses. Trained horses were not evaluated; however, the present findings suggest that sport horses presenting jugular occlusive thrombophlebitis, even unilateral, may be prevented from exercising. Further investigations are necessary to determine the autonomic neurologic activity modulating the hemodynamic changes in the horses with jugular vein occlusion and the mechanisms by which the cardiac variables might differ during exercise.

\section{Acknowledgments}

The authors acknowledge Fundação de Amparo à Pesquisa do Estado de São Paulo (FAPESP) for financial assistance. Poster presented at the 11th International Congress of the World Equine Veterinary Association, Guarujá, Brazil, 2009. 


\section{References}

[1] Cannon JH, Rantanen NW, Grant BD, Keck MT. Jugular venous prosthesis on the horse: a preliminary study. J Equine Vet Sci 1983;3:185-9.

[2] Ettlinger JJ, Palmer JE, Benson C. Bacteria found on intravenous catheters removed from horses. Vet Rec 1992;130:248-9.

[3] Knottenbelt DC, Pascoe RR. Disorders of the cardiovascular system. In: Knottenbelt DC, Pascoe RR, editors. A color atlas of diseases and disorders of the horse. Barcelona: Wolfe; 1994. p. 157-88.

[4] Rijkenhuizen ABM, Van Swieten HA. Reconstruction of the jugular vein in horses with post thrombophlebitis stenosis using saphenous vein graft. Equine Vet J 1998;30:236-9.

[5] Divers TJ. Prevention and treatment of thrombosis, phlebitis and laminitis in horses with gastrointestinal diseases. Vet Clin North Am Equine Pract 2003;19:779-90.

[6] Dolente BA, Beech J, Lindborg S, Smith G. Evaluation of risk factors for development of catheter-associated jugular thrombophlebitis in horses: 50 cases (1993-1998). J Am Vet Med Assoc 2005;227: 1134-41.

[7] Moreau P, Lavoie JP. Evaluation of athletic performance in horses with jugular vein thrombophlebitis: 91 cases (1988-2005). J Am Vet Med Assoc 2009;235:1073-8.

[8] Sellon DC, Wise LN. Disorders of the hematopoietic system. In: Reed SM, Bayly WM, Sellon DC, editors. Equine internal medicine. St. Louis, MO: WB Saunders; 2010. p. 730-76.

[9] Baskurt OK, Farley RA, Meiselman HJ. Erythrocyte aggregation tendency and cellular properties in horse, human, and rat: a comparative study. Am J Physiol 1997;273:H2604-12.

[10] Edens LM. Iatrogenic thrombophlebitis. In: Colahan PT, Mathew IG, Merritt AM, Moore JN, editors. Equine medicine and surgery. St. Louis, MO: Mosby; 1999. p. 416-9.

[11] Lankveld DPK, Ensink JM, Van Dijk P, Klein WR. Factors influencing the occurrence of thrombophlebitis after post-surgical long-term intravenous catheterization of colic horses: a study of 38 cases. J Vet Med A 2001;48:545-52.

[12] Russell TM, Kearney C, Pollock PJ. Surgical treatment of septic jugular thrombophlebitis in nine horses. Vet Surg 2010;39:627-30.

[13] Bonagura JD, Reef VB, Schwarzwald CC. Cardiovascular diseases. In: Reed SM, Bayly WM, Sellon DC, editors. Equine internal medicine. St. Louis, MO: WB Saunders; 2010. p. 372-487.

[14] Guyton AC, Hall JE, editors. Medical physiology. Philadelphia: WB Saunders; 2005.

[15] Evans DL. Cardiovascular adaptations to exercise and training. Vet Clin North Am Equine Pract 1985;1:513-31.

[16] Eaton MD, Evans DL, Hodgson DR, Rose RJ. Maximal accumulated oxygen deficit in Thoroughbred racehorses. J Appl Physiol 1995;78:1564-8.
[17] Bayly WM, Gabel AA, Barr SA. Cardiovascular effects of submaximal aerobic training on a treadmill in Standardbred horses, using a standardized exercise test. Am J Vet Res 1983;44:544-53.

[18] Fazio F, Ferrantelli V, Piccione G, Caola G. Variations in some electrocardiographic parameters in the trotter during racing and training. Vet Res Commun 2003;27(suppl 1):S229-32.

[19] Piccione G, Fazio F, Giudice E, Grasso F, Caola G. Changes in hematological parameters and clotting times in the horse during long distance running. Med Weter 2004;60:587-90.

[20] Piccione G, Fazio F, Giudice E, Grasso F, Caola G. Exercise-induced changes in the clotting times and fibrinolytic activity during official 1600 and 2000 meters trot races in Standardbred horses. Acta Vet Brno 2005;74:509-14.

[21] Piccione G, Grasso F, Fazio F, Giudice E. The effect of physical exercise on the daily rhythm of platelet aggregation and body temperature in horses. Vet J 2008;176:216-20.

[22] Dias DPM, Teixeira LG, Canola PA, Albernaz RM, Marques JA, Lacerda-Neto JC. Long-term facial artery catheter implantation for serial arterial blood sampling and invasive arterial blood pressure measurement in horses. Vet J 2012;192:541-3.

[23] Sirotsky CO, Santiago JM, Almeida FQ, Silva LLF, Miranda ACT, Carrilho SS, et al. Heart rate of event horses during treadmill exercise and cross country phase. In: Proceedings of the XI ABRAVEQ Annual Meeting. São Paulo, Brazil; 2010 [in Portuguese].

[24] Kandel ER, Schwartz JH, Jessell TM, editors. Principles of neural science. New York: McGraw-Hill; 2000.

[25] Comerota AJ, Harwick RD, White JV. Jugular venous reconstruction: a technique to minimize morbidity of bilateral radical neck dissection. J Vasc Surg 1986;3:322-9.

[26] Pearce JMS. Queckenstedt's manoeuvre. J Neurol Neurosurg Psychiatry 2006;77:728.

[27] Barbosa RG, Borghesan AC, Cerqueira NF, Hussni CA, Alves ALG, Nicoletti JLM, et al. Physiopathology of jugular vein thrombosis and thrombophlebitis in horses: review. Vet Zootec 2009;16:26-37 [in Portuguese].

[28] Hamlin RL, Klepinger WL, Gilpin KW, Smith CR. Autonomic control of heart rate in the horse. Am J Physiol 1972;222:976-8.

[29] Durando MM, Reef VB, Birks EK. Right ventricular pressure dynamics during exercise: relationship to stress echocardiography. Equine vet J Suppl 2002;34:472-7.

[30] Poole DC, Erickson HH. Heart and vessels: function during exercise and response to training. In: Hinchcliff KW, Kaneps AJ, Geor RJ, editors. Equine sports medicine and surgery. Philadelphia: WB Saunders; 2004. p. 699-727.

[31] Borell EV, Langbein J, Desprès G, Hansen S, Leterrier C, MarchantForde J, et al. Heart rate variability as a measure of autonomic regulation of cardiac activity for assessing stress and welfare in farm animals-a review. Physiol Behav 2007;92:293-316.

[32] Berne RM, Levy MN. The arterial system. In: Berne RM, Levy MN, Koeppen BM, Stanton BA, editors. Physiology. London: Mosby; 1998. p. $415-28$. 